gemindertes Hunger- und Durstgefühl. Das Selbstvertrauen steigt, die Kontaktfreudigkeit auch. Ebenfalls gesteigert wird das sexuelle Verlangen. Dem gegenüber stehen Hyperthermie, Schwindel, Kreislauf- und Herzrhythmusstörungen, Angstzustände bis hin zu paranoiden Wahnvorstellungen.

So attraktiv die Droge für manchen Konsumenten sein mag - den Süchtigen selbst macht sie nicht schöner. Eine charakteristische Nebenwirkung des starken Amphetaminkonsums ist der "Meth-Mund“. Manchen Zielen des Meth-Gebrauchs - mehr Selbstvertrauen, Kontaktsuche, Steigerung des Sextriebs - ist diese fortgeschrittene Form von Zahnkaries eher abträglich. Härtel-Petri bringt es so auf den Punkt: „Einen solchen Mund will keiner küssen." (methmouthpictures.com).

Nur 20-30\% der Amphetaminabhängigen werden bisher vom Drogenhilfesystem erreicht. Annegret Sievert, leitende Psychologin am Bezirkskrankenhaus Hochstadt, gebraucht dafür den Ausdruck „hidden population“. Sie setzt auf ein neurobiologisch-verhaltenstherapeutisches Konzept, das der stationären Rehabilitation amphetaminabhängiger Patienten dient. Es orientiert sich an Modellen, die ursprünglich für KokainUser in Kalifornien entwickelt worden sind.

Parallel zu einer 24-wöchigen Gruppentherapie, an der auch Konsumenten anderer Drogen teilnehmen, werden den Amphetaminsüchtigen zehn spezifische Sitzungen angeboten. $\mathrm{Zu}$ den Inhalten, die darin behandelt werden, gehören
- Besonderheiten der Substanzen (Neurotoxizität) und deren Konsequenzen für den Rehabilitationsprozess

— Dauer und Umgang mit emotionalen Störungen

_ Antriebsstörungen

_ vermindertes Selbstwertgefühl

_ kognitive Störungen

_ psychotische Störungen, Gefahr von Flashbacks

_ Gewichtszunahme

_ depressive Störungen als Ursache für den Gebrauch von Stimulanzien

_ stimulanzienspezifische Rückfallgefahren

_ Auslöser für Craving

— beziehungslose, gierige Sexualität unter Amphetaminen mit den entsprechenden Rückfallgefahren

_ die Fähigkeit, unter Amphetaminen trotz schwerer sexueller Traumata normale sexuelle Kontakte ausleben zu können.

\section{Vom Wochenendkonsumenten zum Süchtigen} Sievert widerspricht der Ansicht, es handle sich bei den Amphetaminabhängigen praktisch nur um sehr junge Menschen, die auf der Überholspur leben möchten: „Zu den Betroffenen gehören auch 40-Jährige mit einer 60-Stunden-Woche, die am Wochenende noch mithalten möchten, deshalb Amphetamine nehmen und in die Sucht abgleiten." Dr. Robert Bublak

$5^{\text {th }}$ Int. Roundtable: Crystal Speed \& Co., 13. Interdisziplinärer Kongress für Suchtmedizin, München, 7.7.2012

\author{
Nur $20-30 \%$ der \\ Amphetamin- \\ abhängigen \\ werden bisher \\ vom Drogenhilfe- \\ system erreicht.
}

\title{
Neue psychoaktive Substanzen: Auf Wikipedia ist kein Verlass
}

Eigentlich fängt es schon mit den Bezeichnungen an:„Modedrogen“, "Legal Highs“, „Research Chemicals" - für Roland HärtelPetri vom Bezirkskrankenhaus Bayreuth sind dies alles verharmlosende Begriffe. Er spricht stattdessen lieber von „Neuen psychoaktiven Substanzen“ (NPS). Gemeint sind damit synthetische Verbindungen wie Mephedron, Butylon oder MDPV. Verkauft werden sie als Badesalz, Möbelpolitur oder Kakteendünger. Doch zum Waschen, Putzen und Gärtnern werden sie höchstens versehentlich verwendet. Denn sie wirken als amphetaminartige, also stimulierende Drogen - und sie unterliegen hierzulande nicht dem Betäubungsmittelgesetz. Via Internet sind sie problemlos zu beziehen. Unproblematisch sind sie deshalb noch lange nicht. Ein Umstand, den nicht jeder einsehen mag. „Was nicht verboten ist, ist auch nicht gefährlich", zitiert Härtel-Petri eine Patientin, die von den vermeintlich harmlosen Partydrogen in die Sucht getrieben wurde. In einem Land wie unserem, so meinte die junge Frau, würde Gefährliches doch zweifellos aus dem Verkehr gezogen. So schnell, wie neue NPS auf den Markt kommen, kann der Gesetzgeber sie freilich gar nicht verbieten. Härtel-Petri schätzt, dass derzeit 300 bis 500 solcher Drogen zirkulieren.

Ein Problem ist, dass nicht deklariert wird, was das angebliche Badesalz enthält. Und selbst wenn - beim nächste Mal kann sich die Zusammensetzung bereits wieder geändert haben. Den Usern ist das egal; sie verlassen sich darauf, was ihre Freunde von den Tütchen halten. Für Suchtmediziner stellt sich hingegen verschärft die Frage, wie sie hier den Überblick behalten können. Härtel-Petri warnt davor, sich auf die üblichen Informationsquellen wie etwa Wikipedia zu verlassen. Was Drogen angehe, sei das Onlinelexikon "unterwandert". Stattdessen empfiehIt er Adressen, die mit verlässlichen Informationen aufwarten:

http://www.emcdda.europa.eu/publications/drug-profiles/ synthetic-cathinones/de

Auf dieser Seite der Europäischen Beobachtungsstelle für Drogen und Drogensucht finden sich Profile der einzelnen Substanzgruppen.

http://www.psychonautproject.eu/

Seiten des von der EU unterstützten Psychonaut Web Mapping Projects zur Internet-basierten Suche nach Informationen, aus denen sich neue Trends im Drogenkonsum ablesen lassen.

\section{https://rednetproject.eu}

Internetauftritt des „Recreational Drugs European Network"(ReDNet)-Projekts. Es handelt sich dabei laut eigenen Angaben um eine multizentrische Studie zu den NPS mit dem Ziel, ,"den Informationsgehalt zu verbessern, der jungen Menschen (16 bis 24 Jahre) und Mitarbeitern des Gesundheitswesens über diese neuen Drogen und die mit ihrem Konsum potenziell entstehenden Gesundheitsrisiken zur Verfügung steht".

Dr. Robert Bublak 